\title{
Group Consensus with a Dynamic Leader for Multiagent Systems via Sampled-Data Control
}

\author{
Hong Xia, Ting-Zhu Huang, Jin-Liang Shao, and Jun-Yan Yu \\ School of Mathematical Sciences, University of Electronic Science and Technology of China, Chengdu, Sichuan 611731, China \\ Correspondence should be addressed to Hong Xia; xiahong@uestc.edu.cn
}

Received 18 March 2014; Revised 2 June 2014; Accepted 9 June 2014; Published 3 July 2014

Academic Editor: Zbigniew Leśniak

Copyright (C) 2014 Hong Xia et al. This is an open access article distributed under the Creative Commons Attribution License, which permits unrestricted use, distribution, and reproduction in any medium, provided the original work is properly cited.

\begin{abstract}
This paper considers a group consensus problem with a dynamic leader for multiagent systems in a sampled-data setting. With the leader's state available to only a fraction of the followers, a distributed linear protocol based on sampled-data control is proposed for group consensus under fixed directed topology. On basis of $M$-matrix theory, we derive a sufficient condition on the sampling period and the control parameter for ultimate boundedness of the tracking errors. Furthermore, simulation examples are provided to demonstrate the effectiveness of the theoretical results.
\end{abstract}

\section{Introduction}

Over the past few decades, cooperative control and distributed coordination of multiagent systems have attracted increasing attention from many different disciplines, such as mathematics, physics, biology, sociology, and engineering science [1-9]. As a type of critical problems for cooperative control of multiple agents, consensus problem has been an active area of research. In [2], Jadbabaie et al. investigated the consensus of the linearized Vicsek model; they demonstrated that a simple neighbor rule makes all agents eventually move in the same direction under a joint connection condition. Olfati-Saber and Murray [3] established a systematical framework of a consensus problem in continuous-time multiagent systems with fixed and switching topology and communication time-delays. Following this line, numerous results have been reported on consensus of multiagent systems. For details, please refer to [10-13] and the references therein.

A particularly interesting topic is the leader-following consensus problem, whose objective is that the followers track a leader with local interaction. The leader-following consensus problem has been studied from different perspectives (see, e.g., [14-21]). In [14], the authors considered tracking control for first-order consensus with an active leader and gave a local controller together with a neighborbased state-estimation rule. They further extended the results to the case of second-order consensus in [15]. In addition, Ren explored the consensus problem of multiagent systems with respect to a time-varying leader in [16]. In [17], Ren further studied leader-following consensus problem with, respectively, bounded control effort and directed switching interaction topologies. For multiagent systems with multiple leaders, Shi et al. investigated distributed tracking of a convex set specified by multiple leaders with unmeasurable velocities in [18]. It is worth pointing out that the information transmission among all the agents is continuous in [14-21].

In reality, it might be quite difficult or expensive to ensure the continuity of information exchange between agents due to the unreliability of information channels, the finite capability of transmission bandwidth of networks, the limited sensing ability, and the constraints of cost. Thus, sampleddata control for multiagent systems is more coincident with applications. For example, the authors proposed a proportional and derivative like (PD-like) protocol for multiagent systems with a time-varying reference state in [22]. Also, they studied convergence of two distributed sampled-data coordination protocols for double-integrator dynamics under fixed topology in [23]. Furthermore, convergence analyses of two distributed sampled-data coordination algorithms under directed switching topology were addressed in [24]. More results on sampled-data control for multiagent systems can be found in $[25,26]$. 
In contrast to the consensus problems studied in the aforementioned results, group consensus [27, 28] or cluster consensus [29] concerns a multiagent system which is divided into multiple groups, and information exchange exists not only two agents in a group but also in different groups. It aims to design appropriate protocols or algorithms such that agents in a multiagent system reach more than one consistent state, that is, to find some appropriate control inputs such that consensus can be achieved in each group. In fact, group consensus or cluster consensus is a more general concept in comparison with traditional consensus. Moreover, it is suitable for some practical applications because of the complexities of lots of applicable multiagents systems. The authors solved a group average-consensus problem for networks with fixed topologies in [27] and further addressed group consensus in distributed multiagent systems with switching topologies and communication delays in [28]. Moreover, sufficient conditions for group consensus in directed networks were obtained in [29].

Note that the group consensus or cluster consensus problems investigated in [27-29] were all for continuous-time multiagent systems, and they were also leaderless consensus problems. Although group consensus without a leader is useful in many cases, there are many other applications that require a dynamic leader. A common example is formation control, where the followers regulate their states according to their state deviations and attain the expected formation. Inspired by the analysis above, we investigate the group consensus problem with a dynamic leader via sampled-data control. According to specific requirements, the agents in the system are divided into the desired groups, and each group converges to a state which has an expected deviation from that of the leader. By utilizing $M$-matrix theory, we consider the case where the state of leader is available to only a subset of followers. We first give a protocol for continuoustime multiagent systems; then for more realistic applications, we propose a group consensus protocol based on sampleddata control and analyze the convergence of the protocol. Consequently, we obtain the condition on the sampling period and the control parameter to ensure that the tracking errors are ultimately bounded.

\section{Preliminaries}

2.1. Graph and M-Matrix Theory. For a given matrix $A \in$ $\mathbb{R}^{n \times n}$, $\operatorname{det}(A)$ denotes its determinant, $\|A\|_{\infty}$ represents its maximum row sum norm, $\sigma(A)$ is the set of all eigenvalues of $A$, and $\rho(A)$ denotes its spectral radius. A matrix $A$ is said to be positive stable if all of its eigenvalues have positive real parts, and it is Hurwitz stable (in the continuous-time sense) if all of its eigenvalues have negative real parts, while it is Schur stable (in the discrete-time sense) if all of its eigenvalues have magnitude less than $1 . A=\left(a_{i j}\right)_{n \times n}$ is called a nonnegative matrix if $a_{i j} \geq 0, i, j=1, \ldots, n$. $I_{n} \in \mathbb{R}^{n \times n}$ is an $n \times n$ identity matrix and $0_{n \times n} \in \mathbb{R}^{n \times n}$ is an $n \times n$ zero matrix, $1_{n}=[1, \ldots 1]^{T} \in \mathbb{R}^{n}$ and $0_{n}=[0, \ldots 0]^{T} \in \mathbb{R}^{n} .\|x\|_{\infty}$ denotes the max norm of a vector $x \in \mathbb{R}^{n}$. $i$ is the imaginary unit.
Given a complex number $\mu \in \mathbb{C}, \operatorname{Re}(\mu), \operatorname{Im}(\mu)$, and $|\mu|$ are its real part, imaginary part, and modulus, respectively.

A directed graph $\mathscr{G}=(\mathscr{V}, \mathscr{E})$ consists of a vertex set $\mathscr{V}=\{1,2, \ldots, n\}$, an edge set $\mathscr{E} \subseteq \mathscr{V} \times \mathscr{V}$. An edge $(i, j)$ in a weighted directed graph $\mathscr{G}$ denotes that agent $i$ can access the state information of agent $j$ but not necessarily vice versa. The index set of neighbors of node $i$ is denoted by $N_{i}=$ $\{j \in \mathscr{V}:(i, j) \in \mathscr{E}\}$. The weighted adjacency matrix of graph $\mathscr{G}$ is denoted by $A=\left[a_{i j}\right] \in \mathbb{R}^{n \times n}$, where $a_{i j}>0$ if $(i, j) \in \mathscr{E}$ and $a_{i j}=0$ otherwise. Moreover, we assume $a_{i i}=0$ for all $i \in \mathscr{V}$. Let the Laplacian matrix $L=\left[l_{i j}\right] \in \mathbb{R}^{n \times n}$ associated with $A$ be defined as $l_{i i}=\sum_{j=1, j \neq i}^{n} a_{i j}$ and $l_{i j}=-a_{i j}$. It is straightforward to verify that $L$ has at least one zero eigenvalue with a corresponding eigenvector $1_{n}$. A directed path is a sequence of edges in a directed graph of the form $\left(i_{1}, i_{2}\right),\left(i_{2}, i_{3}\right), \ldots$, where $i_{j} \in \mathscr{V}$. If there exists a path from node $i$ to node $j$, we say that $j$ is reachable from $i$.

If each agent is regarded as a vertex, then the interaction topology associated with the agents is conveniently described by a directed graph $\overline{\mathscr{G}}$. In fact, $\overline{\mathscr{G}}$ includes $n$ followers (related to graph $\mathscr{G}$ ) and one leader (labeled as vertex 0 ) with directed edges from some vertices to vertex 0 . The leader adjacency matrix associated with graph $\mathscr{G}$ is defined as a matrix $B=$ $\operatorname{diag}\left\{b_{1}, \ldots, b_{n}\right\}$, where $b_{i}>0$ if vertex 0 is a neighbor of the vertex $i$ and $b_{i}=0$ otherwise. For $\overline{\mathscr{G}}$, if there is a path in $\overline{\mathscr{G}}$ from every vertex $i$ in $\mathscr{G}$ to vertex 0 , we say that vertex 0 is globally reachable in $\overline{\mathscr{G}}$. For convenience, we define $P=$ $\left(p_{i j}\right)_{n \times n}=L+B$ with $p_{i i}=\left(\sum_{j \in N_{i}} a_{i j}+b_{i}\right)$ being the $i$ th diagonal element of the matrix $P$, and $D=\operatorname{diag}\left\{p_{11}, p_{22}, \ldots, p_{n n}\right\} \in$ $\mathbb{R}^{n \times n}$.

$M$-matrix is an important and special class of matrix, which arises in many areas of application. Some definitions and lemmas concerning $M$-matrix are recalled in the following.

Definition 1 (see [30]). Let $Z_{n}=\left\{A=\left(a_{i j}\right)_{n \times n} \in M_{n}(\mathbb{R})\right.$ : $a_{i j} \leq 0$ if $\left.i \neq j, i, j=1,2, \ldots n\right\}$, where $M_{n}(\mathbb{R})$ denotes the set of all $n \times n$ matrices with entries from $\mathbb{R}$. Then a matrix $A=\left(a_{i j}\right)_{n \times n}$ is called an $M$-matrix if $A \in Z_{n}$ and $A$ is positive stable.

Lemma 2 (see [31]). If $A=\left(a_{i j}\right)_{n \times n} \in Z_{n}$, the following statements are equivalent:

(i) A is positive stable; that is, $A$ is an M-matrix;

(ii) $A$ is nonsingular and $A^{-1}$ is a nonnegative matrix;

(iii) The diagonal entries of $A$ are positive, and $\rho(B)<1$, where $B=I_{n}-X^{-1} A, X=\operatorname{diag}\left\{a_{11}, a_{22}, \ldots, a_{n n}\right\}$.

Lemma 3 (see [26]). $P=L+B$ is an $M$-matrix if and only if the vertex 0 is globally reachable in $\overline{\mathscr{G}}$.

The following lemmas will be useful in the analysis of the convergence of the sampled-data protocol.

Lemma 4 (see [30]). If $\rho(A)<1, A \in M_{n}(\mathbb{R})$, then the series $I+A+A^{2}+\cdots$ converges to the sum $\left(I_{n}-A\right)^{-1}$. 
Lemma 5 (Schur's formula [32]). Let $A, B, C, D \in \mathbb{R}^{n \times n}$ and $M=\left(\begin{array}{ll}A & B \\ C & D\end{array}\right)$. Then $\operatorname{det}(M)=\operatorname{det}(A D-B C)$, if $A, B, C$ and $D$ commute pairwise.

Lemma 6 (see [33]). Given a complex-coefficient polynomial

$$
f(s)=s^{2}+(a+i b) s+c+i d
$$

where $a, b, c, d \in \mathbb{R}, f(s)$ is Hurwitz stable if and only if $a>0$ and $a b d+a^{2} c-d^{2}>0$.

2.2. Model Description. Consider a system consisting of $n$ followers and one leader, where the $n$ followers are separated into $m(m \leq n)$ groups. Let $E_{q}, q=1,2, \ldots, m$ be the set of all the followers in the $q$ th group. Our objective is to design suitable protocol or algorithm to realize group consensus, in the presence of information exchanges between followers of different groups.

All the followers move with the first-order dynamics, described by

$$
\dot{x}_{i}(t)=u_{i}(t), \quad i=1,2, \ldots, n,
$$

where $x_{i}(t) \in \mathbb{R}^{k}$ represents the state of the follower $i$ and $u_{i}(t) \in \mathbb{R}^{k}$ is the control input, called protocol or algorithm, to be designed based on the local information received by follower $i$ from its neighbors.

The motion of the leader is independent, expressed as

$$
\dot{x}_{0}(t)=f(t)
$$

where $f(t)$ is bounded, piecewise continuous in $t$. Here we assume that all agents are in a one-dimensional space $(k=$ 1) for the simplicity of presentation. However, all results hereafter are still valid for the $k$-dimensional $(k>1)$ case by introduction of the Kronecker product.

Definition 7. Group consensus with a leader is said to be achieved asymptotically if the states of followers satisfy $\lim _{t \rightarrow \infty}\left\|x_{i}(t)-x_{0}(t)-\omega_{q}(t)\right\|=0$, where $x_{0}(t)$ is the state of the leader and $\omega_{q}(t)$ denotes the desired deviation between the leader and the followers in the $q$ th group for $q=1,2, \ldots, m$.

Different from [27-29], we introduce a leader in the group consensus problem of this paper, and the followers in the network can be divided into several groups upon specific request. Furthermore, the followers in a group converge to a state which has an expected relative deviation from the state of the leader.

\section{Sampled-Data Group Consensus with a Dynamic Leader}

3.1. Sampled-Data Group Consensus Protocol. Denote the desired deviation between the leader and the followers in the $q$ th group as $\omega_{q}(t)$; we then propose the following continuous-time protocol:

$$
\begin{gathered}
u_{i}(t)=\dot{\omega}_{q}(t) \\
+\frac{1}{p_{i i}} \sum_{s=1}^{m} \sum_{j \in E_{s}} a_{i j}\left[\dot{x}_{j}(t)-\dot{\omega}_{s}(t)\right. \\
\quad-\alpha\left(\left(x_{i}(t)-x_{j}(t)\right)\right. \\
\left.\left.\quad-\left(\omega_{q}(t)-\omega_{s}(t)\right)\right)\right] \\
+\frac{b_{i}}{p_{i i}}\left[\dot{x}_{0}(t)-\alpha\left(x_{i}(t)-\omega_{q}(t)-x_{0}(t)\right)\right] \\
i \in E_{q}, \quad q=1,2, \ldots, m,
\end{gathered}
$$

where $a_{i j}$ is the $(i, j)$ th entry of the adjacency matrix $A, b_{i}$ is the $i$ th diagonal element of the leader adjacency matrix $B, \alpha>$ 0 is the control parameter, and $p_{i i}=\left(\sum_{j \in N_{i}} a_{i j}+b_{i}\right)$ is the $i$ th diagonal element of the matrix $P=L+B$.

Lemma 8. Protocol (4) solves a group consensus problem asymptotically if and only if the vertex 0 is globally reachable in $\overline{\mathscr{G}}$.

Proof. Noticing that $P$ is an $M$-matrix, it follows from part (iii) of Lemma 2 that $p_{i i}>0$, which implies that protocol (4) is well defined. Let $\bar{x}(t)=\left(\bar{x}_{1}(t), \bar{x}_{2}(t), \ldots, \bar{x}_{n}(t)\right)^{T}$, where $\bar{x}_{i}(t)=x_{i}(t)-x_{0}(t)-\omega_{q}(t), i \in E_{q}, q=1,2, \ldots, m$. Then using the fact that $\dot{x}_{j}(t)=u_{j}(t)$, protocol (4) can be written as

$$
\begin{aligned}
p_{i i} \dot{\bar{x}}_{i}(t) & -\sum_{s=1}^{m} \sum_{j \in E_{s}} a_{i j} \dot{\bar{x}}_{j}(t) \\
= & -\alpha\left[\sum_{s=1}^{m} \sum_{j \in E_{s}} a_{i j}\left(\bar{x}_{i}(t)-\bar{x}_{j}(t)\right)+b_{i} \bar{x}_{i}(t)\right],
\end{aligned}
$$

which can be expressed in matrix form as

$$
P \dot{\bar{x}}(t)=-\alpha P \bar{x}(t)
$$

Note that $P$ is invertible since $P$ is an $M$-matrix; it is clear that there exists a unique solution for $\bar{x}_{i}(t)(i=1,2, \ldots, n)$. In fact, the solution for $(6)$ is $\bar{x}(t)=e^{-\alpha t} \bar{x}(0)$. Therefore, $\bar{x}(t)$ converges to $0_{n}$ asymptotically; that is, $x_{i}(t) \rightarrow x_{0}(t)+\omega_{q}(t)$ as $t \rightarrow \infty, i \in E_{q}, q=1,2, \ldots, m$.

Remark 9. Note that $u_{i}(t), i=1,2, \ldots, n$, in (4) depends not only on the information states of its neighbors but also on their derivatives. In the special case that $\omega_{q}(t) \equiv 0$, for $q=$ $1,2, \ldots, m$, protocol (4) is equivalent to protocol (3.4) in [10].

Note that each agent's information control input in protocol (4) requires the instantaneous measurements of its neighbors' states and their derivatives. However, it may be difficult 
to implement the requirement in practice; we are hence motivated to consider a sampled-data setting, where each agent can only communicate with its neighbours at discrete sampling instants. With the sampling period $T$, the control input $u_{i}(t)$ holds as

$$
u_{i}(t)=u_{i}[k], \quad k T \leq t \leq(k+1) T,
$$

where $k$ is the discrete-time index and $u_{i}[k]$ denotes the control input at $t=k T$. Discretizing (2) with zero-order hold, one obtains the dynamics of each follower as follows:

$$
x_{i}[k+1]=x_{i}[k]+T u_{i}[k], \quad i=1, \ldots, n,
$$

where $x_{i}[k+1]$ and $x_{i}[k]$ denote, respectively, the position of follower $i$ at $t=(k+1) T$ and $t=k T$. The sampled-data protocol on basis of protocol (4) is then presented as follows:

$$
\begin{aligned}
& u_{i}[k] \\
& =\frac{\omega_{q}[k]-\omega_{q}[k-1]}{T} \\
& +\frac{1}{p_{i i}} \sum_{s=1}^{m} \sum_{j \in E_{s}} a_{i j}\left[\frac{x_{j}[k]-x_{j}[k-1]}{T}\right. \\
& -\frac{\omega_{s}[k]-\omega_{s}[k-1]}{T} \\
& -\alpha\left(\left(x_{i}[k]-x_{j}[k]\right)\right. \\
& \left.\left.-\left(\omega_{q}[k]-\omega_{s}[k]\right)\right)\right] \\
& +\frac{b_{i}}{p_{i i}}\left[\frac{x_{0}[k]-x_{0}[k-1]}{T}\right. \\
& \left.-\alpha\left(x_{i}[k]-x_{0}[k]-\omega_{q}[k]\right)\right], \\
& i \in E_{q}, \quad q=1,2, \ldots, m,
\end{aligned}
$$

where $\dot{\omega}_{q}(t)$ and $\dot{x}_{j}(t)$ in $(4)$ at $t=k T$ are approximated by $\left(\omega_{q}[k]-\omega_{q}[k-1]\right) / T$ and $\left(x_{j}[k]-x_{j}[k-1]\right) / T$, respectively, $a_{i j}$ is the $(i, j)$ th entry of the adjacency matrix $A, b_{i}$ is the $i$ th diagonal element of the leader adjacency matrix $B$, and $p_{i i}$ is the $i$ th diagonal element of the matrix $P$.

3.2. Convergence Analysis of the Sampled-Data Protocol. In this subsection, we focus on the convergence analysis of the sampled-data group consensus protocol with the availability of the leader's state to some followers. Define the tracking error for follower $i$ as $\varepsilon_{i}[k]=x_{i}[k]-x_{0}[k]-\omega_{q}[k], g_{i}[k]=$ $2\left(x_{0}[k]+\omega_{q}[k]\right)-x_{0}[k-1]-\omega_{q}[k-1]-x_{0}[k+1]-\omega_{q}[k+1]$, $i \in E_{q}, q=1,2, \ldots, m$, then (8) using (9) can be written as

$$
\begin{aligned}
\varepsilon_{i}[k+1]= & \varepsilon_{i}[k] \\
+ & \frac{1}{p_{i i}} \sum_{j=1}^{n} a_{i j}\left(\varepsilon_{j}[k]-\varepsilon_{j}[k-1]\right. \\
& \left.\quad-\alpha T\left(\varepsilon_{i}[k]-\varepsilon_{j}[k]\right)\right) \\
& -\frac{\alpha T b_{i}}{p_{i i}} \varepsilon_{i}[k]+g_{i}[k],
\end{aligned}
$$

which can be equally written as

$$
\begin{aligned}
\varepsilon[k+1]= & {\left[(1-\alpha T) I_{n}+(1+\alpha T) D^{-1} A\right] \varepsilon[k] } \\
& -D^{-1} A \varepsilon[k-1]+g[k]
\end{aligned}
$$

where $\varepsilon[k]=\left(\varepsilon_{1}[k], \ldots, \varepsilon_{n}[k]\right)^{T}, g[k]=\left(g_{1}[k], \ldots, g_{n}[k]\right)^{T}$, $D=\operatorname{diag}\left\{p_{11}, p_{22}, \ldots, p_{n n}\right\} \in \mathbb{R}^{n \times n}$ is the degree matrix of $\overline{\mathscr{G}}$ and $A$ is the adjacency matrix associated with graph $\mathscr{G}$. Note that

$$
A=D-L-B=D-P
$$

thus

$$
\begin{gathered}
D^{-1} A=I_{n}-D^{-1} P, \\
(1-\alpha T) I_{n}+(1+\alpha T) D^{-1} A=2 I_{n}-(1+\alpha T) D^{-1} P .
\end{gathered}
$$

As a result, (11) can be written in matrix form as

$$
Z[k+1]=H Z[k]+F g[k],
$$

where $Z[k+1]=(\varepsilon[k+1], \varepsilon[k])^{T}, F=\left(I_{n}, 0_{n \times n}\right)^{T}$, and

$$
H=\left(\begin{array}{cc}
2 I_{n}-(1+\alpha T) D^{-1} P & -\left(I_{n}-D^{-1} P\right) \\
I_{n} & 0_{n \times n}
\end{array}\right) .
$$

It follows that the solution of (14) is

$$
Z[k+1]=H^{k} Z[0]+\sum_{j=0}^{k-1} H^{k-(j+1)} F g[j] .
$$

It can be noted from (16) that the eigenvalues of $H$ play an important role in the convergence analysis. As a result, we study the eigenvalues of matrix $H$ before giving our main results.

Lemma 10. Suppose that vertex 0 is globally reachable in $\overline{\mathscr{G}}$;

(i) then $\rho\left(I_{n}-D^{-1} P\right)<1$, where $P=L+B$, $L$ and $B$ are the Laplacian matrix and the leader adjacency matrix of graph $\mathscr{G}$, respectively, and $D=\operatorname{diag}\left\{p_{11}, p_{22}, \ldots, p_{n n}\right\}$;

(ii) let $\mu_{j}=\xi_{j}+i \eta_{j}, j=1,2, \ldots, n$, be the $j$ th eigenvalue of $D^{-1} P$, where $\xi_{j}=\operatorname{Re}\left(\mu_{j}\right), \eta_{j}=\operatorname{Im}\left(\mu_{j}\right)$; then

$$
\lambda_{j}=\frac{\left(4 \xi_{j}-2 \xi_{j}^{2}-2 \eta_{j}^{2}\right)\left(\xi_{j}^{2}+\eta_{j}^{2}\right)}{4 \eta_{j}^{2}+\left(\xi_{j}^{2}+\eta_{j}^{2}\right)^{2}}>0
$$

holds; 
(iii) $\rho(H)<1$ if and only if the control parameter $\alpha$ and sampling period $T$ satisfy

$$
\alpha T<\min _{j=1, \ldots, n} \lambda_{j}
$$

Proof. When vertex 0 is globally reachable in $\overline{\mathscr{G}}$, it follows, from Lemma 3, that the matrix $P=L+B$ is an $M$-matrix. Then according to Lemma $2, \rho\left(I_{n}-D^{-1} P\right)<1$ holds.

To show part (ii), note that $D^{-1} P \in Z_{n}$ and $\left(D^{-1} P\right)^{-1}=$ $P^{-1} D$ is nonnegative. Thus, by Lemma $2, D^{-1} P$ is an $M$ matrix, which implies that $D^{-1} P$ is positive stable; that is, $\xi_{j}>0$. Consequently, $4 \eta_{j}^{2}+\left(\xi_{j}^{2}+\eta_{j}^{2}\right)^{2}>0$ and $\xi_{j}^{2}+\eta_{j}^{2}>0$. On the other hand, it follows from part (i) that $\rho\left(I_{n}-D^{-1} P\right)<1$. Equally, $\left|1-\mu_{j}\right|<1$ for $\forall \mu_{j} \in \sigma\left(D^{-1} P\right)$. Then, by some manipulation, we can obtain that $\left(2 \xi_{j}-\xi_{j}^{2}-\eta_{j}^{2}\right)>0$. As a result, $\lambda_{j}>0$ holds.

For the part (iii), let $y$ be an eigenvalue of $H$; then the characteristic polynomial of $H$ is given by

$$
\begin{aligned}
& \operatorname{det}\left(y I_{2 n}-H\right) \\
& =\operatorname{det}\left(\left[\begin{array}{cc}
y I_{n}-\left[2 I_{n}-(1+\alpha T) D^{-1} P\right] & I_{n}-D^{-1} P \\
-I_{n} & y I_{n}
\end{array}\right]\right) \\
& =\operatorname{det}\left(y^{2} I_{n}-\left[2 I_{n}-(1+\alpha T) D^{-1} P\right] y I_{n}+I_{n}-D^{-1} P\right) \\
& =\prod_{j=1}^{n}\left\{y^{2}-\left[2-(1+\alpha T) \mu_{j}\right] y+1-\mu_{j}\right\},
\end{aligned}
$$

where we have used Schur's formula. Therefore, the eigenvalues of $H$ satisfy

$$
y^{2}-\left(2-(1+\alpha T) \mu_{j}\right) y+1-\mu_{j}=0 .
$$

It may be complicated to determine directly whether the roots of (20) are within the unit circle. Instead, we apply the bilinear transformation $y=(s+1) /(s-1)$ to $(20)$ to yield

$$
\alpha T \mu_{j} s^{2}+2 \mu_{j} s+(2+\alpha T)\left(1-\mu_{j}\right)+2-\alpha T=0
$$

that is,

$$
\alpha T \mu_{j}\left[s^{2}+\frac{2}{\alpha T} s+\frac{4-(2+\alpha T) \mu_{j}}{\alpha T \mu_{j}}\right]=0 .
$$

Note that the bilinear transformation maps the interior of the unit circle one-to-one onto the open left half plane. Consequently, the roots of (20) are within the unit circle if and only if

$$
s^{2}+\frac{2}{\alpha T} s+\frac{4-(2+\alpha T) \mu_{j}}{\alpha T \mu_{j}}
$$

is Hurwitz stable. Then, for $\forall \mu_{j} \in \sigma\left(D^{-1} P\right)$, set

$$
\begin{gathered}
a=\frac{2}{\alpha T}, \quad b=0, \\
c=\frac{\left(4-2 \xi_{j}-\alpha T \xi_{j}\right) \xi_{j}-\left(2 \eta_{j}+\alpha T \eta_{j}\right) \eta_{j}}{\alpha T\left(\xi_{j}^{2}+\eta_{j}^{2}\right)}, \\
d=\frac{-4 \eta_{j}}{\alpha T\left(\xi_{j}^{2}+\eta_{j}^{2}\right)} .
\end{gathered}
$$

According to Lemma 6, (23) is Hurwitz stable if and only if

$$
\begin{gathered}
\frac{2}{\alpha T}>0, \\
\frac{\left(4-2 \xi_{j}-2 \alpha T \xi_{j}\right) \xi_{j}-\left(2 \eta_{j}+\alpha T \eta_{j}\right) \eta_{j}}{\alpha T}>\frac{4 \eta_{j}^{2}}{\xi_{j}^{2}+\eta_{j}^{2}},
\end{gathered}
$$

which leads to

$$
\alpha T<\frac{\left(4 \xi_{j}-2 \xi_{j}^{2}-2 \eta_{j}^{2}\right)\left(\xi_{j}^{2}+\eta_{j}^{2}\right)}{4 \eta_{j}^{2}+\left(\xi_{j}^{2}+\eta_{j}^{2}\right)^{2}} .
$$

Therefore, all eigenvalues of $H$ are within the unit circle if and only if the control parameter $\alpha$ and sampling period $T$ satisfy (18).

Remark 11. In [11], Ren has proved that $\rho\left(D^{-1} A\right)<1$ if the leader has directed paths to all followers in $\overline{\mathscr{G}}$ (Lemma 8.1 in [11]). Note that $D^{-1} A=D^{-1}(D-P)=I_{n}-D^{-1} P$. Therefore, part (i) of Lemma 10 can be regarded as a simple proof of it using $M$-matrix theory.

Based on Lemma 10, we now present the main result.

Theorem 12. Suppose that vertex 0 is globally reachable in $\overline{\mathscr{G}}$ and there exist positive constants $l_{1}, l_{2}$ such that $\mid x_{0}[k]-x_{0}[k-$ $1] \mid \leq l_{1} T$ and $\left|\omega_{q}[k]-\omega_{q}[k-1]\right| \leq l_{2} T, q=1,2, \ldots, m$, If control parameter $\alpha$ and the sampling period $T$ satisfy (26); then with the sampled-data protocol (9), group consensus can be achieved. In addition,

$$
\lim _{k \rightarrow \infty}\|Z[k]\|_{\infty} \leq 2\left(l_{1}+l_{2}\right) T\left\|\left(I_{2 n}-H\right)^{-1}\right\|_{\infty} .
$$

Proof. Since $\left|x_{0}[k]-x_{0}[k-1]\right| \leq l_{1} T$ and $\left|\omega_{q}[k]-\omega_{q}[k-1]\right| \leq$ $l_{2} T$ for all $k$, we have that $\left|g_{i}[j]\right|=\mid 2\left(x_{0}[j]+\omega_{q}[j]\right)-x_{0}[j-$ $1]-\omega_{q}[j-1]-x_{0}[j+1]-\omega_{q}[j+1] \mid \leq 2\left(l_{1}+l_{2}\right) T, i \in E_{q}, q=$ $1,2, \ldots, m$. Therefore, $\|g[j]\|_{\infty}=\left\|\left(g_{1}[j], \ldots, g_{n}[k]\right)^{T}\right\|_{\infty} \leq$ $2\left(l_{1}+l_{2}\right) T$. It then follows from (16) that

$$
\begin{aligned}
\|Z[k]\|_{\infty} \leq & \left\|H^{k} Z[0]\right\|_{\infty}+\left\|\sum_{j=0}^{k-1} H^{k-(j+1)} F g[j]\right\|_{\infty} \\
\leq & \left\|H^{k}\right\|_{\infty}\|Z[0]\|_{\infty}+2\left(l_{1}+l_{2}\right) T \\
& \times\left\|\sum_{j=0}^{k-1} H^{k-(j+1)}\right\|_{\infty}\|F\|_{\infty} .
\end{aligned}
$$




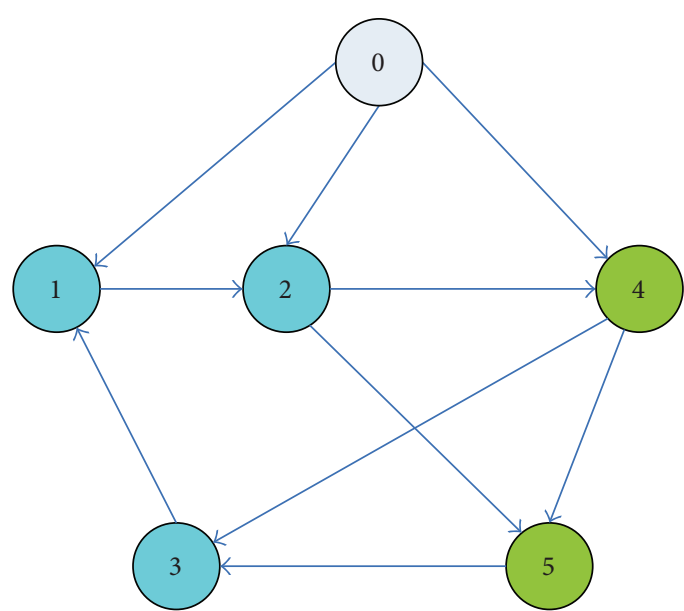

(a) $\overline{\mathscr{G}}_{1}$

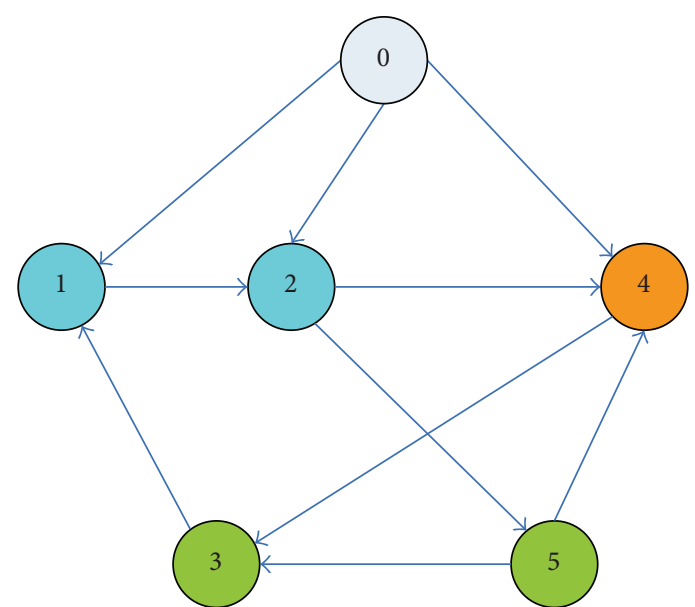

(b) $\overline{\mathscr{G}}_{2}$

FIgURE 1: Interaction topologies $\overline{\mathscr{G}}_{1}$ and $\overline{\mathscr{G}}_{2}$.

By Lemma 10, we have $\rho(H)<1$ and $\lim _{k \rightarrow \infty} H^{k}=0$. It follows from Lemma 4 that $\lim _{k \rightarrow \infty} \sum_{j=0}^{k-1} H^{k-(j+1)}=$ $\left(I_{n}-H\right)^{-1}$. We finally obtain that the tracking errors $x_{i}[k]-$ $x_{0}[k]-\omega_{q}[k]\left(i \in E_{q}, q=1, \ldots, m\right)$ are ultimately bounded by $2\left(l_{1}+l_{2}\right) T\left\|\left(I_{n}-H\right)^{-1}\right\|_{\infty}$. Furthermore, the tracking errors will converge to 0 as $T \rightarrow 0$.

\section{Numerical Simulations}

In this section, several examples are provided to show the effectiveness of our theoretical result. Consider the interaction topologies shown in Figure 1; note that Figure 1(a) and Figure 1(b) have the same topologies where the five followers in Figure 1(a) are separated into two groups: $E_{1}=\{1,2,3\}$ and $E_{2}=\{4,5\}$, while the five followers in Figure 1(b) are separated into three groups: $E_{1}=\{1,2\}, E_{2}=\{3,5\}$, and $E_{3}=\{4\}$. Obviously, vertex 0 is globally reachable in Figure 1. For convenience, the weights of interaction topology $\overline{\mathscr{G}}$ are supposed to be 1 . Then the matrixes $P$ and $D$ are given by

$$
\begin{gathered}
P=\left(\begin{array}{ccccc}
2 & 0 & -1 & 0 & 0 \\
-1 & 2 & 0 & 0 & 0 \\
0 & 0 & 2 & -1 & -1 \\
0 & -1 & 0 & 3 & -1 \\
0 & -1 & 0 & 0 & 1
\end{array}\right) \\
D=\left(\begin{array}{lllll}
2 & 0 & 0 & 0 & 0 \\
0 & 2 & 0 & 0 & 0 \\
0 & 0 & 2 & 0 & 0 \\
0 & 0 & 0 & 3 & 0 \\
0 & 0 & 0 & 0 & 1
\end{array}\right)
\end{gathered}
$$

where $L$ and $B$ are the Laplacian matrix and the leader adjacency matrix, respectively. The eigenvalues of $D^{-1} P$ are $\mu_{1}=0.3097, \mu_{2}=0.9424+0.6520 i, \mu_{3}=0.9424-0.6520 i$, $\mu_{4}=1.5489$, and $\mu_{5}=1.2567$.
For the sampled-data protocol (9), we let the position of the leader, the relative state deviation between $E_{1}$ and the leader, and the relative state deviation between $E_{2}$ and the leader be $x_{0}[k]=k T, \omega_{1}[k]=\sin [k T]$, and $\omega_{2}[k]=\cos [k T]$, respectively. In addition, the relative state deviation between $E_{3}$ and the leader in Figure $1(\mathrm{~b})$ is chosen as $\omega_{3}[k]=$ $\tanh [k T]$. Furthermore, we assume that the initial positions of the followers are $x_{i}[-1]=(0,0,0,0,0)^{T}, i=1, \ldots, 5$ and $x_{i}[0]=(2,-1,1,-2,1)^{T}, i=1, \ldots, 5$. Calculate

$$
\min _{j=1, \ldots, n} \frac{\left(4 \xi_{j}-2 \xi_{j}^{2}-2 \eta_{j}^{2}\right)\left(\xi_{j}^{2}+\eta_{j}^{2}\right)}{4 \eta_{j}^{2}+\left(\xi_{j}^{2}+\eta_{j}^{2}\right)^{2}}=0.4383
$$

Then, according to Theorem 12, the multiagent system can achieve group consensus if $\alpha T<0.4383$. Figure 2(a) and Figure 3(a) show that the tracking errors are bounded with $\alpha=8.6, T=0.05$, and $\alpha=1.4, T=0.3$, respectively. It can be seen that the tracking errors in Figure 3(a) are larger than those in Figure 2(a), which coincide with Theorem 12 since the tracking errors are proportional to the sampling period $T$. Finally, Figure 2(b) shows that the tracking errors become unbounded when $\alpha=0.9, T=0.5$, the same as when $\alpha=2.2, T=0.2$ illustrated by Figure 3(b).

For the continuous-time protocol (4), assume that all the agents move on a plane, namely, $k=2$. Consider the interaction topology depicted in Figure 4; note that $\overline{\mathscr{G}}_{1}, \overline{\mathscr{G}}_{2}$, and $\overline{\mathscr{G}}_{3}$ have indeed the same interaction topologies but different groups. The five followers in Figure 4 are divided into five groups; that is, $m=5$ and $E_{q}=\{q\}, q=$ $1,2, \ldots, 5$. Taking $x_{0}(t)=(t, 0)^{T}$ and $\omega_{q}(t)=(\cos (\pi t / 30+$ $2 q \pi / 5), \sin (\pi t / 30+2 q \pi / 5))^{T}, q=1,2, \ldots, 5$, Figure 5 shows the trajectories of agents, where the initial positions of agents are randomly generated in a given bounded region. It can 


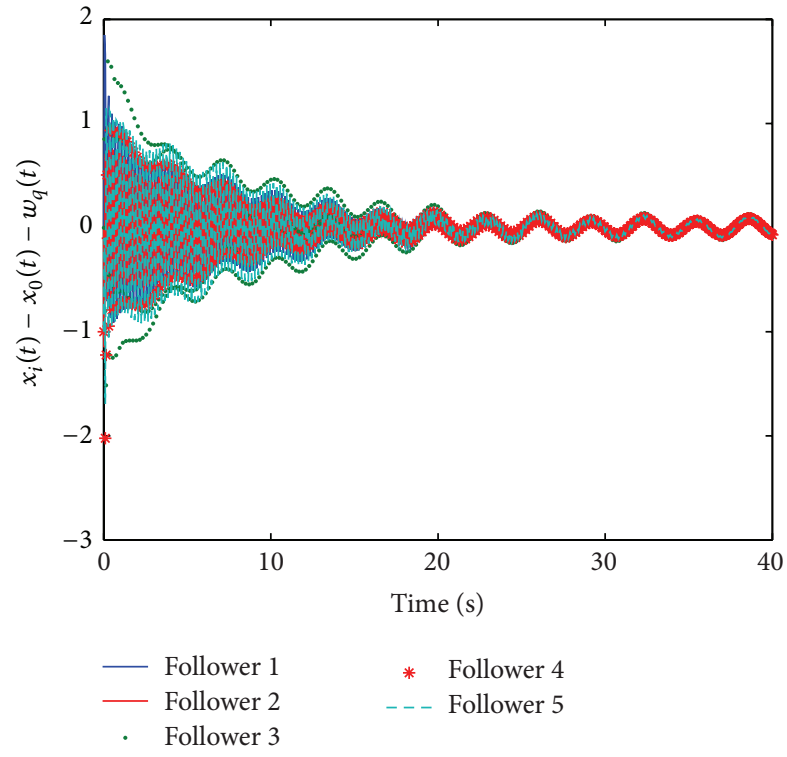

(a) Tracking errors $(\alpha=8.6, T=0.05)$

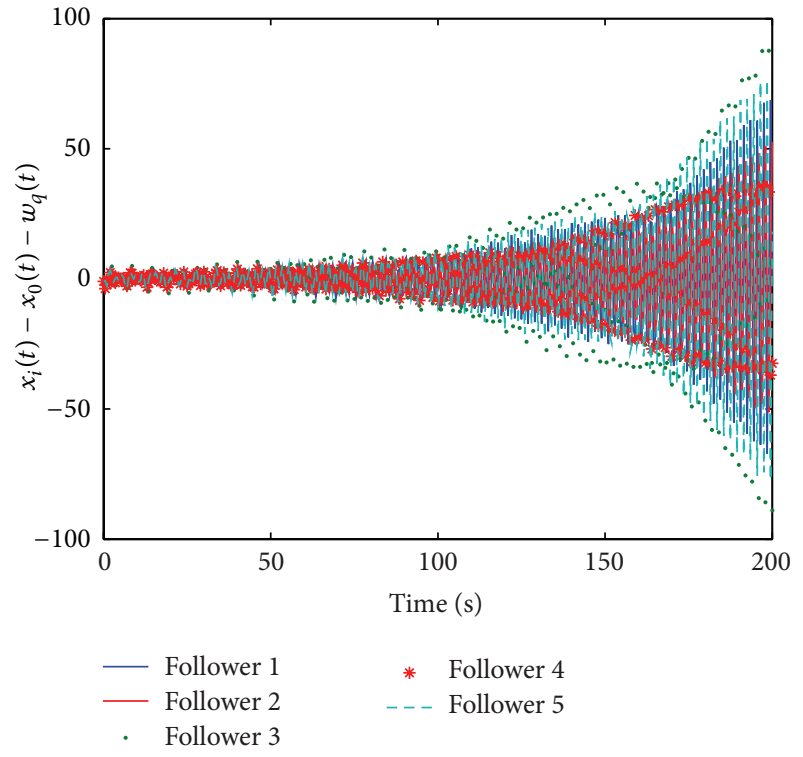

(b) Tracking errors $(\alpha=0.9, T=0.5)$

FIGURE 2: Tracking errors using the sampled-data protocol (9).

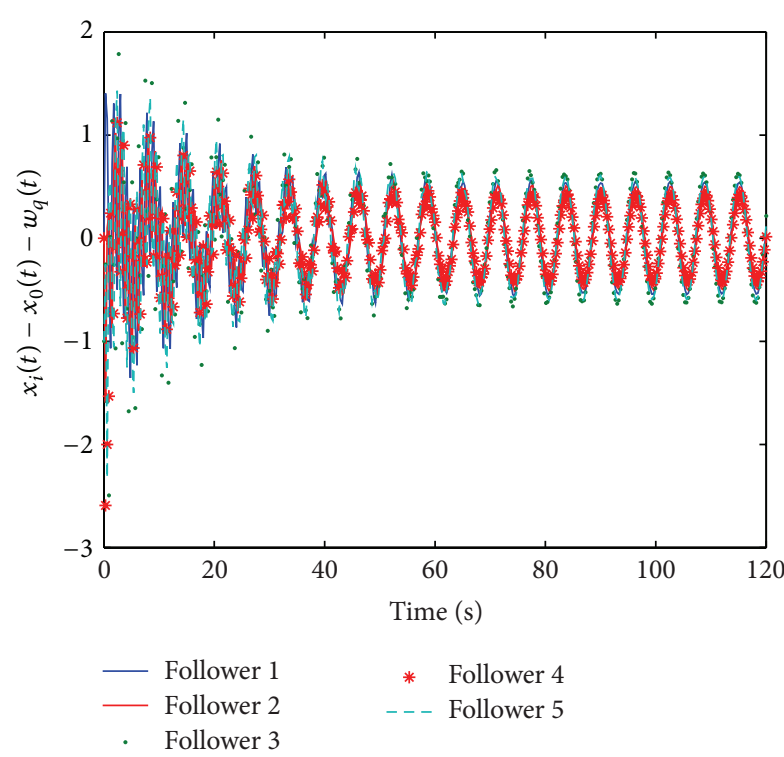

(a) Tracking errors $(\alpha=1.4, T=0.3)$

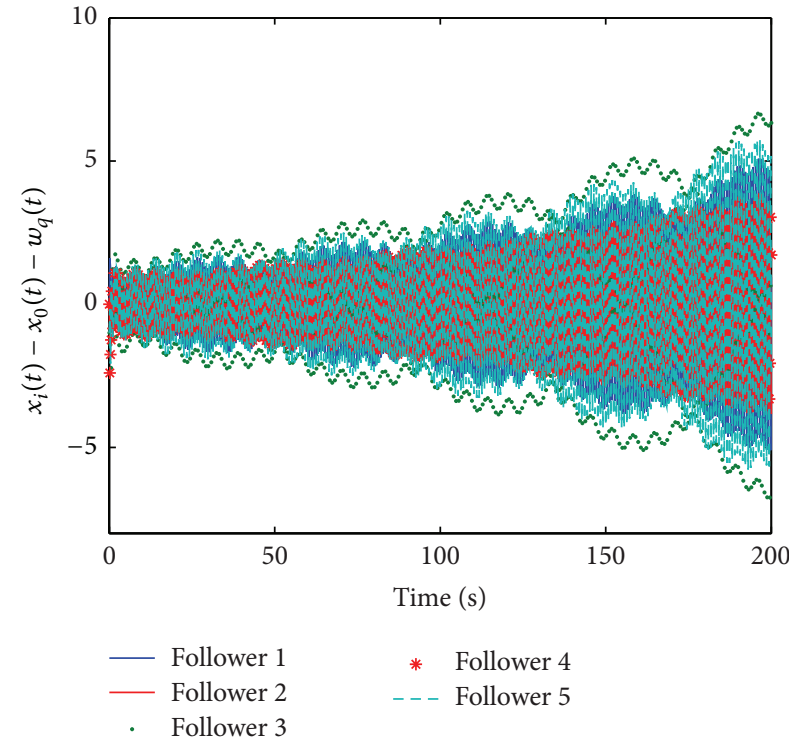

(b) Tracking errors $(\alpha=2.2, T=0.2)$

FIGURE 3: Tracking errors using the sampled-data protocol (9).

be seen that the five followers converge to different states; specially, the lines connecting them form a pentagon.

\section{Conclusion}

In this paper, the group consensus problem with a dynamic leader for multiagent systems in a sampled-data setting is investigated. We propose a distributed coordination protocol based on sampled-data control, in which only a subset of the followers has access to the state of leader. Then, by utilizing $M$-matrix theory, we present the convergence analysis of the protocol. The condition on the sampling period and the control parameter is presented to ensure convergence, and quantitative bounds of the tracking errors are given. Finally, numerical simulations are performed to validate the theoretical results. Our future work will focus on investigating group consensus problem with a leader under a directed switching communication graph. 


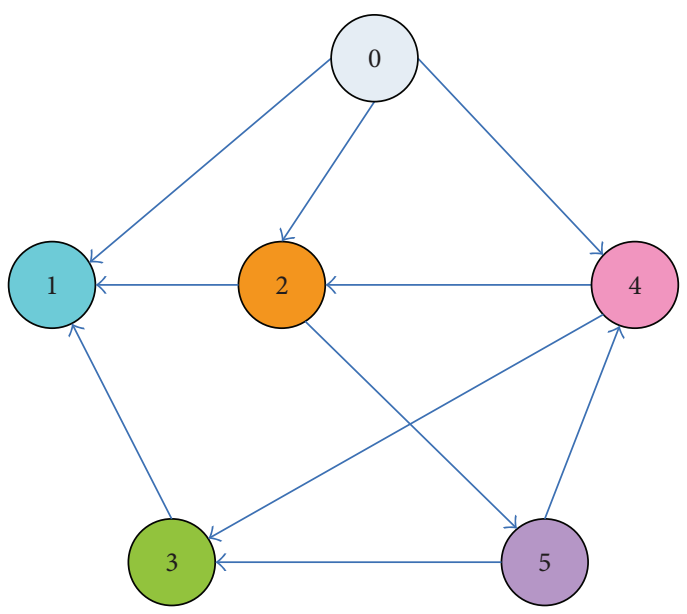

FIGURE 4: Interaction topology $\overline{\mathscr{G}}_{3}$.

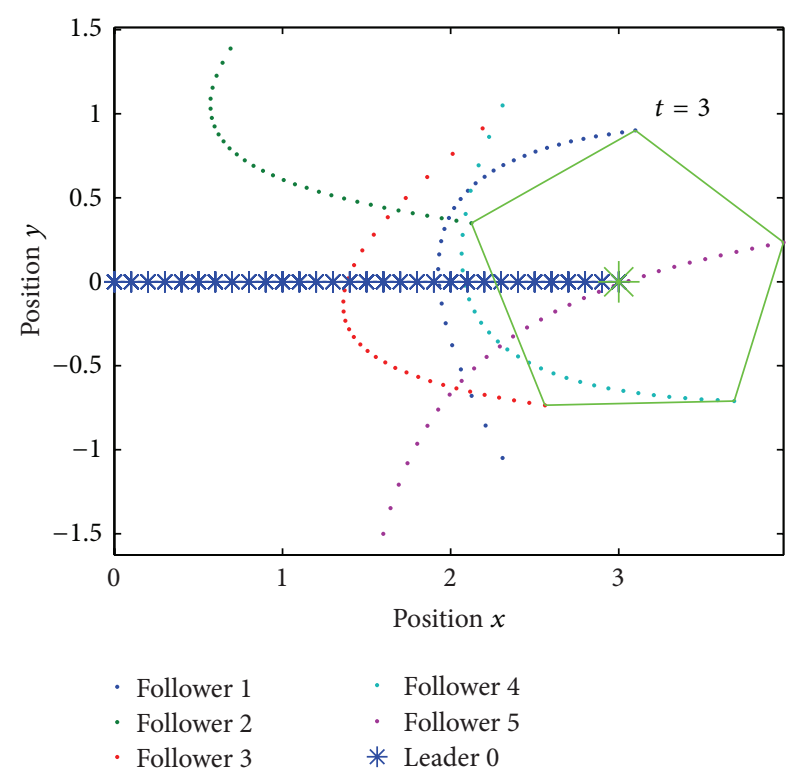

FIGURE 5: The trajectories of agents using the continuous-time protocol (4).

\section{Conflict of Interests}

The authors declare that there is no conflict of interests regarding the publication of this paper.

\section{Acknowledgments}

This research is supported by NSFC (61104141, 61370147, 61170309, and 61301052), Chinese Universities Specialized Research Fund for the Doctoral Program (20110185110020), NSFC Tianyuan foundation (11126104), and the Fundamental Research Funds for the Central Universities (ZYGX2010J106).

\section{References}

[1] T. Vicsek, A. Czirk, E. Ben-Jacob, I. Cohen, and O. Shochet, "Novel type of phase transition in a system of self-driven particles," Physical Review Letters, vol. 75, no. 6, pp. 1226-1229, 1995.

[2] A. Jadbabaie, J. Lin, and A. S. Morse, "Coordination of groups of mobile autonomous agents using nearest neighbor rules," IEEE Transactions on Automatic Control, vol. 48, no. 6, pp. 988-1001, 2003.

[3] R. Olfati-Saber and R. M. Murray, "Consensus problems in networks of agents with switching topology and time-delays," IEEE Transactions on Automatic Control, vol. 49, no. 9, pp. 15201533, 2004.

[4] R. W. Beard and V. Stepanyan, "Synchronization of information in distributed multiple vehicle coordination control," in Proceedings of the 42nd IEEE Conference on Decision and Control (CDC '03), pp. 2029-2034, December 2003.

[5] R. Olfati-Saber and J. S. Shamma, "Consensus filters for sensor networks and distributed sensor fusion," in Proceedings of the 44th IEEE Conference on Decision and Control and European Control Conference (CDC-ECC '05), pp. 6698-6703, Seville, Spain, December 2005.

[6] R. Olfati-Saber, "Flocking for multi-agent dynamic systems: algorithms and theory," IEEE Transactions on Automatic Control, vol. 51, no. 3, pp. 401-420, 2006.

[7] P. Lin and Y. Jia, "Average consensus in networks of multi-agents with both switching topology and coupling time-delay," Physica A, vol. 387, no. 1, pp. 303-313, 2008.

[8] P. Lin and Y. Jia, "Consensus of a class of second-order multiagent systems with time-delay and jointly-connected topologies," IEEE Transactions on Automatic Control, vol. 55, no. 3, pp. 778-784, 2010.

[9] F. Xiao, L. Wang, J. Chen, and Y. Gao, "Finite-time formation control for multi-agent systems," Automatica, vol. 45, no. 11, pp. 2606-2611, 2009.

[10] W. Ren and R. W. Beard, Distributed Consensus in Multi-Vehicle Cooperative Control, Springer, London, UK, 2008.

[11] W. Ren and Y. Cao, Distributed Coordination of Multi-Agent Networks, Springer, London, UK, 2011.

[12] P. Lin and W. Ren, "Constrained consensus in unbalanced networks with communication delays," IEEE Transactions on Automatic Control, vol. 59, no. 3, pp. 775-781, 2014.

[13] P. Lin and W. Ren, "Distributed constrained consensus in the presence of unbalanced switching graphs and communication delays," in Proceedings of the 51st IEEE Conference on Decision and Control (CDC '12), pp. 2238-2243, Maui, Hawaii, USA, December 2012.

[14] Y. Hong, J. Hu, and L. Gao, "Tracking control for multiagent consensus with an active leader and variable topology," Automatica, vol. 42, no. 7, pp. 1177-1182, 2006.

[15] Y. Hong, G. Chen, and L. Bushnell, "Distributed observers design for leader-following control of multi-agent networks," Automatica, vol. 44, no. 3, pp. 846-850, 2008.

[16] W. Ren, "Multi-vehicle consensus with a time-varying reference state," Systems \& Control Letters, vol. 56, no. 7-8, pp. 474-483, 2007.

[17] W. Ren, "Consensus tracking under directed interaction topologies: algorithms and experiments," IEEE Transactions on Control Systems Technology, vol. 18, no. 1, pp. 230-237, 2010. 
[18] H. Xia, T. Huang, J. Shao, and J. Yu, "Second-order leaderfollowing consensus of multiagent systems with time delays," Mathematical Problems in Engineering, vol. 2013, Article ID 505434, 8 pages, 2013.

[19] J. Hu and G. Feng, "Distributed tracking control of leaderfollower multi-agent systems under noisy measurement," Automatica, vol. 46, no. 8, pp. 1382-1387, 2010.

[20] H. Xia, T. Huang, J. Shao, and J. Yu, "Formation control of second-order multi-agent systems with time-varying delays," Mathematical Problems in Engineering, vol. 2014, Article ID 764580, 8 pages, 2014.

[21] H. Xia, T. Huang, J. Shao, and J. Yu, "Leader-following formation control for second-order multi-agent systems with timevarying delays," Transactions of the Institute of Measurement and Control, vol. 36, no. 5, pp. 627-636, 2014.

[22] Y. Cao, W. Ren, and Y. Li, "Distributed discrete-time coordinated tracking with a time-varying reference state and limited communication," Automatica, vol. 45, no. 5, pp. 1299-1305, 2009.

[23] Y. Cao and W. Ren, "Multi-vehicle coordination for doubleintegrator dynamics under fixed undirected/directed interaction in a sampled-data setting," International Journal of Robust and Nonlinear Control, vol. 20, no. 9, pp. 987-1000, 2010.

[24] Y. Cao and W. Ren, "Sampled-data discrete-time coordination algorithms for double-integrator dynamics under dynamic directed interaction," International Journal of Control, vol. 83, no. 3, pp. 506-515, 2010.

[25] Y. Gao and L. Wang, "Consensus of multiple dynamic agents with sampled information," IET Control Theory and Applications, vol. 4, no. 6, pp. 945-956, 2010.

[26] Z. Tang, T. Huang, J. Shao, and J. Hu, "Leader-following consensus for multi-agent systems via sampled-data control," IET Control Theory \& Applications, vol. 5, no. 14, pp. 1658-1665, 2011.

[27] J. Yu and L. Wang, "Group consensus of multi-agent systems with undirected communication graphs," in Proceedings of the 7th Asian Control Conference (ASCC '09), pp. 105-110, August 2009.

[28] J. Yu and L. Wang, "Group consensus in multi-agent systems with switching topologies and communication delays," Systems \& Control Letters, vol. 59, no. 6, pp. 340-348, 2010.

[29] X. Lu, A. Francis, and S. Chen, "Cluster consensus of nonlinearly coupled multi-agent systems in directed graphs," Chinese Physics Letters, vol. 27, no. 5, Article ID 050503, 2010.

[30] R. A. Horn and C. R. Johnson, Topics in Matrix Analysis, Cambridge University Press, New York, NY, USA, 1994.

[31] S. Richard, Matrix Iterative Anaysis, Springer, Berlin, Germany, 2006.

[32] F. R. Gantmakher, The Theory of Matrices, Chelsea, New York, NY, USA, 2000.

[33] P. C. Parks and V. Hahn, Stability Theory, Prentice-Hall, Upper Saddle River, NJ, USA, 1992. 


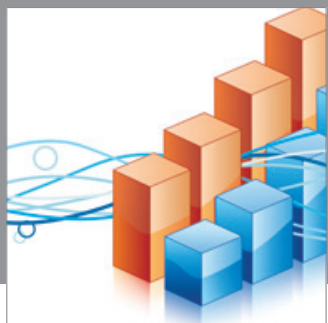

Advances in

Operations Research

mansans

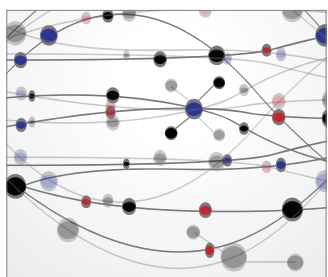

The Scientific World Journal
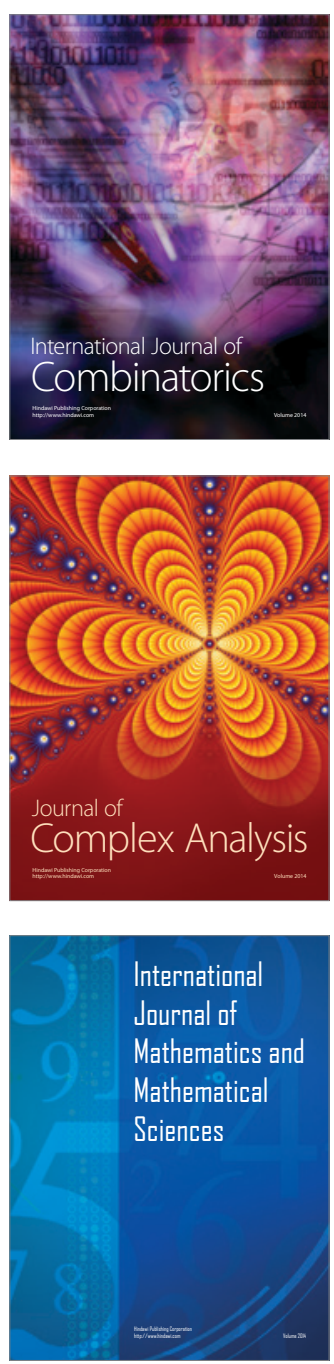
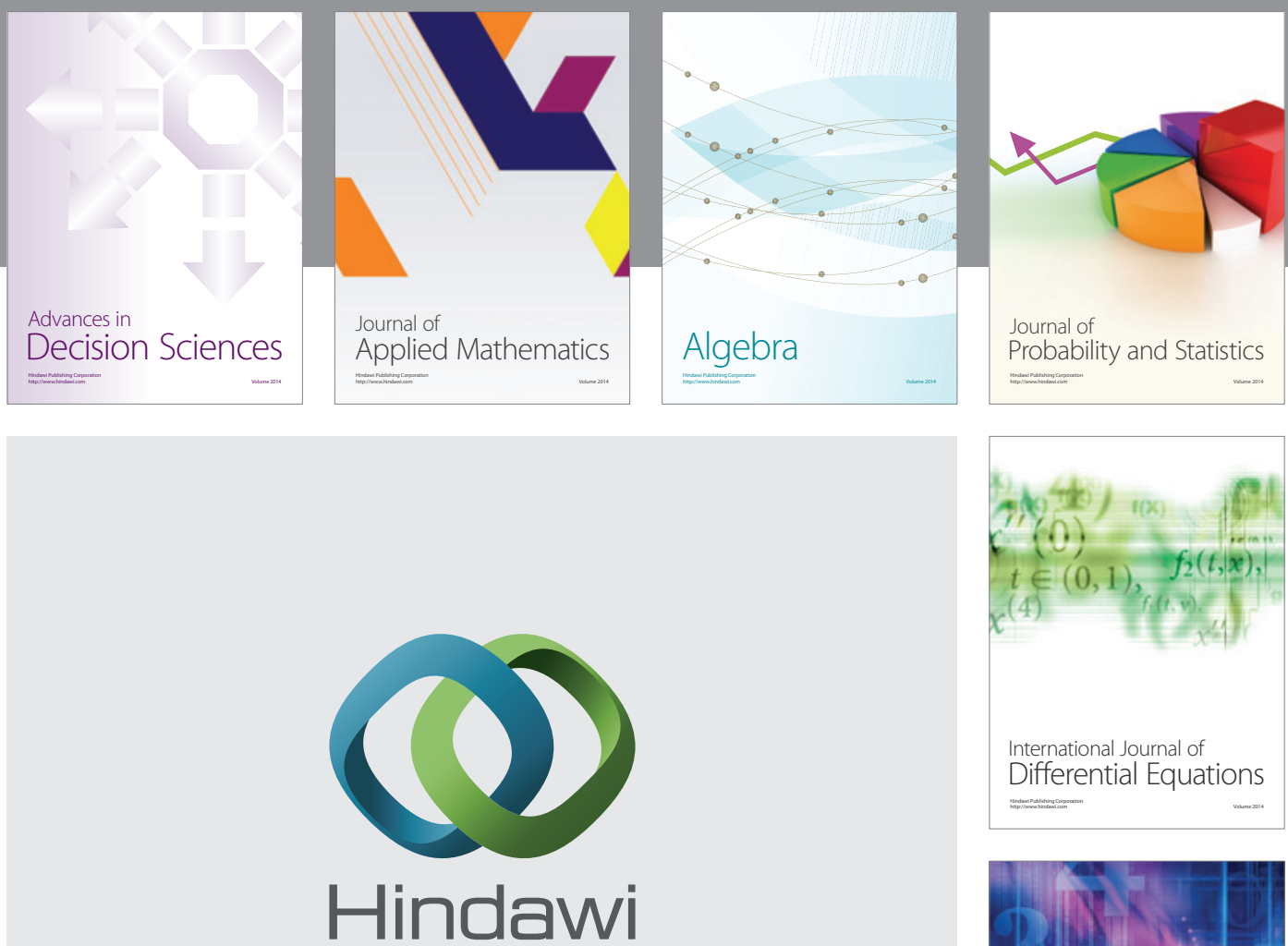

Submit your manuscripts at http://www.hindawi.com
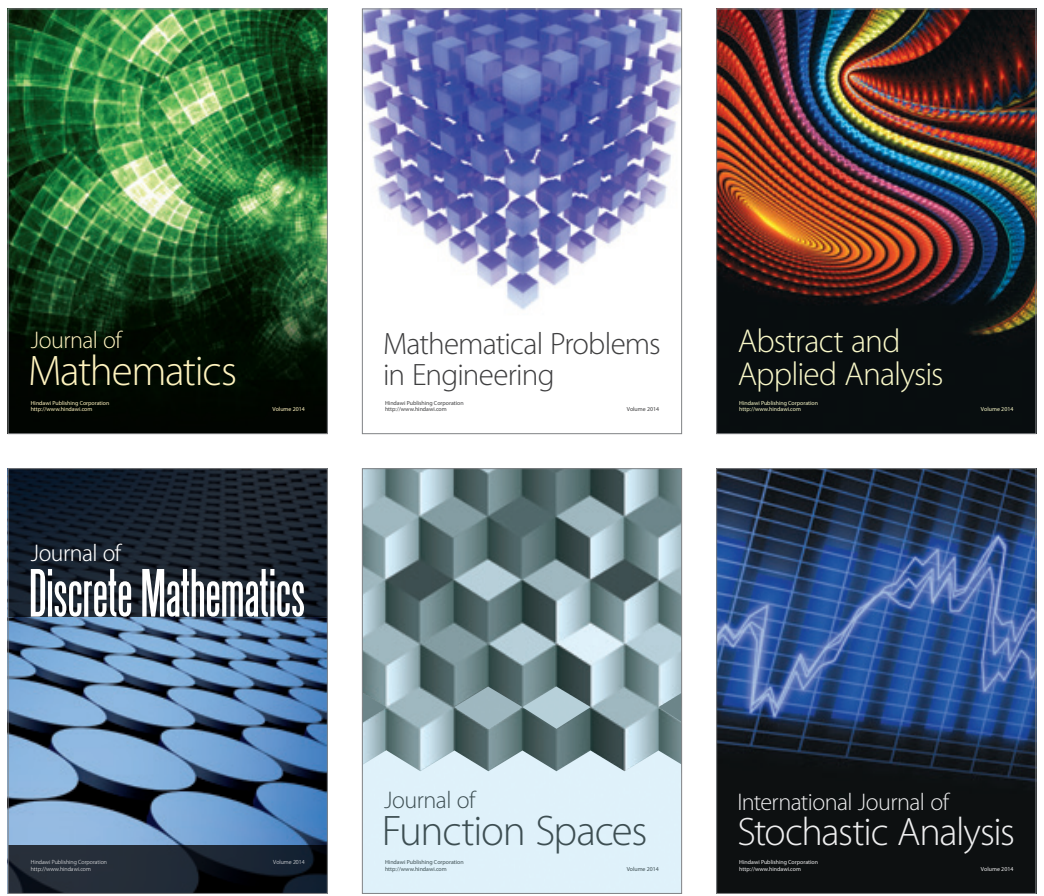

Journal of

Function Spaces

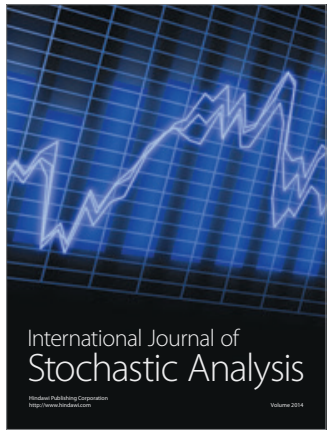

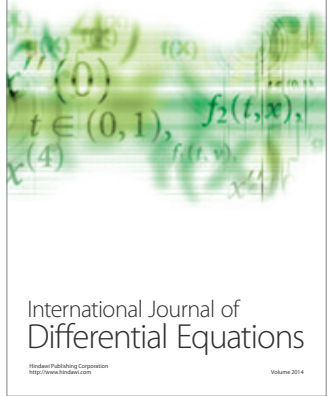
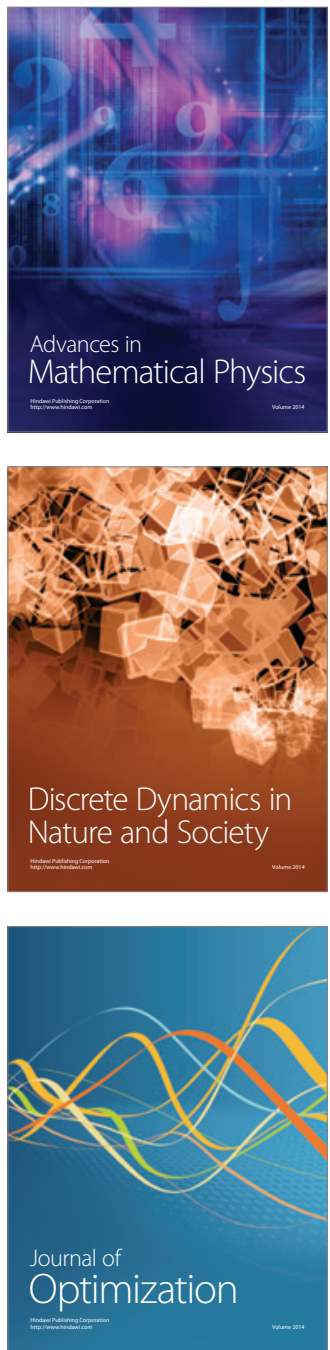